\title{
Characterization of the anisotropy of rough surfaces: Crossing statistics
}

M. Ghasemi Nezhadhaghighi, S. M. S. Movahed, T. Yasseri, and S. Mehdi Vaez Allaei

Citation: Journal of Applied Physics 122, 085302 (2017); doi: 10.1063/1.4998436

View online: https://doi.org/10.1063/1.4998436

View Table of Contents: http://aip.scitation.org/toc/jap/122/8

Published by the American Institute of Physics

\section{Articles you may be interested in}

Electron energy dissipation model of gate dielectric progressive breakdown in $n$ - and $p$-channel field effect transistors

Journal of Applied Physics 122, 085701 (2017); 10.1063/1.4985794

Thermal energy conversion using near-field thermophotovoltaic device composed of a thin-film tungsten radiator and a thin-film silicon cell

Journal of Applied Physics 122, 084302 (2017); 10.1063/1.4989870

Transmission of oxygen radicals through free-standing single-layer and multilayer silicon-nitride and silicondioxide films

Journal of Applied Physics 122, 084101 (2017); 10.1063/1.5000135

A reconfigurable silicon-on-insulator diode with tunable electrostatic doping

Journal of Applied Physics 122, 084502 (2017); 10.1063/1.4999314

Antiferromagnetic anisotropy determination by spin Hall magnetoresistance

Journal of Applied Physics 122, 083907 (2017); 10.1063/1.4986372

Photo-assisted Kelvin probe force microscopy investigation of three dimensional GaN structures with various crystal facets, doping types, and wavelengths of illumination

Journal of Applied Physics 122, 085307 (2017); 10.1063/1.5000137

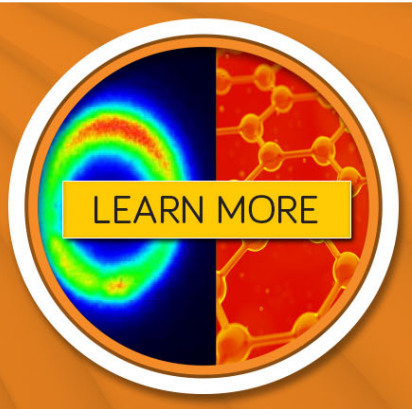




\title{
Characterization of the anisotropy of rough surfaces: Crossing statistics
}

\author{
M. Ghasemi Nezhadhaghighi, ${ }^{1}$ S. M. S. Movahed, ${ }^{2,3, a)}$ T. Yasseri, ${ }^{4,5}$ \\ and S. Mehdi Vaez Allaei ${ }^{6,7, b)}$ \\ ${ }^{1}$ Department of Physics, College of Sciences, Shiraz University, Shiraz 71454, Iran \\ ${ }^{2}$ Department of Physics, Shahid Beheshti University, G.C., Evin, Tehran 19839, Iran \\ ${ }^{3}$ School of Physics, Institute for Research in Fundamental Sciences (IPM), P.O. Box 19395-5531, Tehran, Iran \\ ${ }^{4}$ Oxford Internet Institute, University of Oxford, 1 St Giles', Oxford OX1 3JS, United Kingdom \\ ${ }^{5}$ Alan Turing Institute, 96 Euston Rd., London NW1 2DB, United Kingdom \\ ${ }^{6}$ Department of Physics, University of Tehran, Tehran 14395-547, Iran \\ ${ }^{7}$ The Abdus Salam International Centre for Theoretical Physics, Strada Costiera 11, I-34013 Trieste, Italy
}

(Received 25 February 2017; accepted 31 July 2017; published online 23 August 2017)

\begin{abstract}
In this paper, we propose the use of crossing statistics and its generalizations as a new framework to characterize the anisotropy of a $2 \mathrm{D}$ rough surface. The proposed method is expandable to higher dimensions. By measuring the number of up-crossing, $\nu^{+}$[crossing points with a positive slope at a given threshold of height $(\alpha)$ ], and the generalized roughness function, $N_{\text {tot }}$, it is possible to characterize the nature of an anisotropy, rotational invariance, and Gaussianity of any given surface. In the case of anisotropic correlated self- or multi-affine surfaces, even with different correlation lengths in different directions and/or directional scaling exponents, we examine the relationship between $\nu^{+}$and $N_{\text {tot }}$, and corresponding scaling parameters analytically. The method identifies the direction of anisotropy through the systematic use of P-value statistics. After applying the common methods in determining the corresponding scaling exponents in the identified anisotropic directions, we are able to determine the type and the ratio of the involved correlation lengths. To demonstrate capability and accuracy of the method, as well as to validate the analytical calculations, we apply the proposed measures on synthetic stochastic rough interfaces and rough interfaces generated from the simulation of ion etching. There is a good agreement between analytical results and the outcomes of the numerical models. The proposed algorithm can be implemented through a simple software in various instruments, such as AFM and STM, for surface analysis and characterization. Published by AIP Publishing. [http://dx.doi.org/10.1063/1.4998436]
\end{abstract}

\section{INTRODUCTION}

Isotropy and anisotropy are important characteristics of a given surface or an interface that depends on various conditions and factors. The method of the creation (crack, ${ }^{1}$ growth, ${ }^{2}$ etching ${ }^{3}$ ) and the building blocks of the media can influence the symmetries of the given surface/interface. For instance, in the case of growth via evaporation/condensation, different mechanisms can alter the isotropy of the surface to various extent $^{4-6}$ that can be described by the Anisotropic KardarParisi-Zhang (AKPZ) equation. ${ }^{7-9}$ Different properties of a given rough surface or interface such as friction, diffusivity of particles, wettability, liquid contact angle, and conductivity can be influenced by topography of it. Therefore, adequate characterization of the local (Geometrical) and global (Topological) properties of the surface plays a crucial role in its specifications.

To characterize the anisotropic features of a surface/ interface, it is not enough to only determine the anisotropy direction. The anisotropy can be further characterized by its associated correlation length and/or scaling exponent for systems, which exhibit scaling behavior. ${ }^{4-6}$ Such characterization should be performed however within a universal

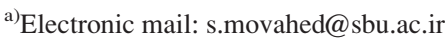

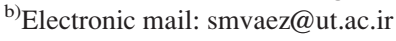

framework, allowing for standardized comparison between surfaces. This becomes even more important in the case of stochastic rough interfaces, where anisotropic features could be screened by the random nature of the surface fluctuations, introducing additional and/or spurious properties. Examples of this situation can be found in the growth of anisotropic organic thin films, or erosion and growth with incident angle. ${ }^{3,10}$

Usual methods to detect anisotropies, e.g., Fourier transform, encounter numerical and technical limits, especially in situations, where having large number of samples to create an adequate statistical ensemble is not possible. Among quantitative methods that can characterize anisotropy, ${ }^{11-17}$ one notable example is the one proposed by Zhao et al. based on light diffraction from anisotropic rough surfaces. ${ }^{4,5}$ Vivo et al. have used the height power spectral density analysis to examine the scaling anisotropic rough surface. ${ }^{18,19}$ Recently, Guillemot et al. have introduced a regularity parameter to quantify the degree of anisotropy of periodic structures on rough surfaces. ${ }^{20}$ Several methods are based on field renormalization $^{19,21,22}$ and a well-known approach is the heightheight correlation function measurement followed by the analysis of directional dependency of the roughness exponents. ${ }^{18,22}$ Even though previous research provides appropriate tools to find the direction of anisotropy, in very few of 
them the possibility of shedding lights on the nature of the anisotropy is provided.

In this paper, we introduce and apply crossing statistics as a measure for characterizing anisotropic feature of a given surface. The proposed approach is independent from the mechanism used to generate the surface, for instance, by erosion or growth, and acceptability works for both self-affine and nonself-affine rough surfaces. We show that this method enables us to quantify the existence of anisotropy and to discriminate isotropic and anisotropic patterns in the real space. From the computational point of view, it can be mounted on the experimental devices, such as atomic force microscopy (AFM) and scanning tunneling microscopy (STM).

The rest of the paper is organized as follows. In Sec. II, we provide a brief explanation on the synthetic generation of isotropic and anisotropic rough surfaces. We introduce the crossing statistics to investigate the height fluctuation of isotropic and anisotropic rough surfaces in Sec. III. Simulations of isotropic and anisotropic rough surfaces and analysis based on crossing statistics by means of theoretical and numerical computation are given in detail in Sec. IV. Finally, summary and conclusions are presented in Sec. V.

\section{SYNTHETIC ISOTROPIC AND ANISOTROPIC ROUGH SURFACES}

In order to demonstrate the affordances of crossing statistics to characterize an anisotropic rough surface, we use two different methods for preparing synthetic rough surfaces. First, we utilize fractional Brownian motion ( $\mathrm{fBm}$ ) for generating synthetic self-affine rough surfaces, associated with a wide range of growth models. Second, a Kinetic Monte Carlo (KMC) method is exploited to model the pattern formation by ion-beam sputtering (IBS). ${ }^{10,23-27}$ These two types represent a wide range of surfaces generated by nanoscale topography in surface growth and erosion processes up to large scale self-affine rough surfaces in macroscopic system sizes. ${ }^{2,28}$ Here, we outline the two methods and introduce the model parameters that control the anisotropy of the generated surfaces.

\section{A. Self-affine surfaces}

Several methods have been introduced for generating synthetic 2D rough surfaces: multiplicative cascading process, ${ }^{29-32}$ random measure $\beta$-model, ${ }^{33} \alpha$-model, ${ }^{34}$ log-stable models, log-infinitely divisible cascade models, ${ }^{35,36}$ and regeneration. ${ }^{37}$ In addition, the successive random addition method, ${ }^{38}$ the Weierstrass-Mandelbrot function, ${ }^{39}$ the optimization method, ${ }^{40}$ and oriented non-Gaussian method ${ }^{41}$ have been introduced and implemented in computational surface generators. Another efficient way of generating a rough surface is the modified Fourier filtering method. ${ }^{42}$

To generate a Gaussian anisotropic rough surface in 2D with a given anisotropic correlation lengths, the following power spectrum is considered: ${ }^{4}$

$$
S^{(2 \mathrm{D})}(\mathbf{k})=\frac{4 \pi \gamma \sigma_{0}^{2} k_{c}^{2 \gamma} \xi_{u} \xi_{w}}{L^{2}\left[k_{c}^{2}+\xi_{u}^{2} k_{u}^{2}+\xi_{w}^{2} k_{w}^{2}\right]^{\gamma+1}},
$$

where $\xi_{u}$ and $\xi_{w}$ are correlation lengths in $u$ and $w$ directions as an orthogonal set on the surface respectively. $\mathbf{k}:\left(k_{u}, k_{w}\right)$ is the wave vector, $k_{c}$ is the cutoff wave vector, and $\gamma$ is the scaling exponent. The variance of surface height is represented by $\sigma_{0}$, and $L$ is the size of the rough surface. For scaling anisotropic model, we use the following power spectrum: ${ }^{4}$

$$
S^{(2 \mathrm{D})}(\mathbf{k})=\frac{4 \pi \sigma_{0}^{2} k_{c}^{2\left(\gamma_{u}+\gamma_{w}\right)} \xi_{u} \xi_{w} \frac{\Gamma\left(\frac{1}{2}+\gamma_{u}\right)}{\Gamma\left(\gamma_{u}\right)} \frac{\Gamma\left(\frac{1}{2}+\gamma_{w}\right)}{\Gamma\left(\gamma_{w}\right)}}{L^{2}\left[k_{c}^{2}+\xi_{u}^{2} k_{u}^{2}\right]^{\gamma_{u}+1 / 2}\left[k_{c}^{2}+\xi_{w}^{2} k_{w}^{2}\right]^{\gamma_{w}+1 / 2}},
$$

where $\gamma_{u}$ and $\gamma_{w}$ are the scaling exponents in directions $u$ and $w$, respectively. Both power spectra [Eqs. (1) and (2)] represent the fractional Brownian motion. The heights of two points on the surface separated with distance $r<1 / k_{c}$ are correlated, whereas correlation is diminished for $r>1 / k_{c}{ }^{1,43-45}$ Moreover, Eqs. (1) and (2) imply $\sigma_{0}^{2}=\left(\frac{L}{2 \pi}\right)^{2} \int d \mathbf{k} S^{(2 \mathrm{D})}(\mathbf{k})$.

\section{B. Anisotropic pattern in surface erosion}

Surface sputtering by energetic ions $\left(\mathrm{Ne}^{+}, \mathrm{Ar}^{+}, \mathrm{Xe}^{+}\right.$, etc.) as an efficient method to manufacture nano-scale structures on surface of solids (glass, metals, semiconductors, etc.) is widely applied and examined in the last five decades. ${ }^{46-48}$

The base of an Ion-Beam Sputtering (IBS) experiment is shooting energetic ions in the range of $\mathrm{keV}$ towards a prepared surface of solid. Etching the surface due to atomic collision cascades initiated by the energetic ions along with enhanced surface diffusion of lateral ad-atoms leads to formation of regular patterns with typical size of $10-100 \mathrm{~nm}$, reported in both experiments and computer simulations. ${ }^{48-50}$ Nano-ripples, quantum dots, and nano-holes with symmetric and amorphous lateral distributions are among different types of patterns forming in IBS experiments.

Though such patterns are highly desirable for practical and technological applications in many different areas, ${ }^{51}$ there is not much known about the underlying mechanisms of formation and anomalous features of them. Coarsening of the patterns in time, presence of symmetries in unexpected directions, and complete phase diagram of type of the patterns forming in different experimental conditions are the most important and puzzling challenges in theoretical understanding of the phenomena as well as in experimental investigations.

The Monte Carlo modeling set-up, which is based on the theoretical model of Bradley-Harper, ${ }^{3}$ includes two main parts: erosion of the surface atoms due to collisions of the ions and the diffusion of lateral atoms of the solid, enhanced by the heat released by collision cascades. We consider a 3D cubic lattice of atoms of $L \times L$ substrate size, with periodic boundary conditions and solid-on-solid restriction (see Fig. 1). Ions navigate to the surface from random starting points at a plane parallel to the initially flat solid surface (i.e., $(x-y)$ plane). As indicated in Fig. 1, an ion beam follows a straight trajectory that makes an angle $\theta$ with the normal of this plane. The projection of the ion-beam direction on the plane target surface $[(x-y)$ plane $]$ makes an azimuthal angle of $\phi_{\exp }$ relative to the $x$ axis. All ions penetrate into the bulk for a typical distance and release their energy modeled by a 3D Gaussian 


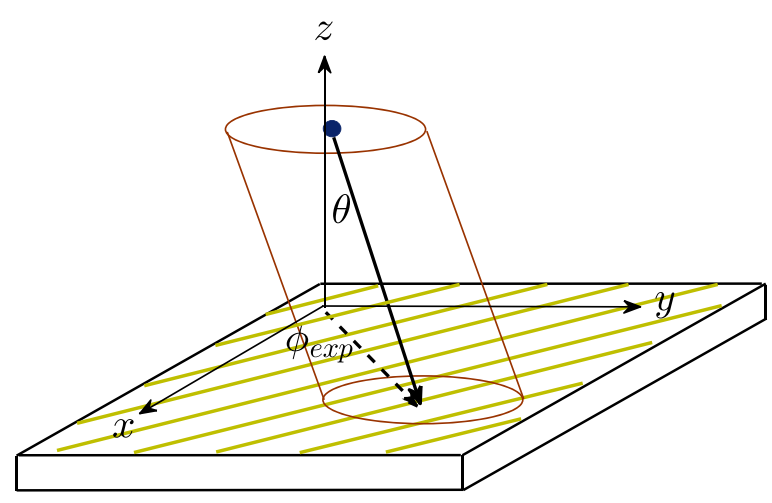

FIG. 1. A sketch showing the Monte Carlo modeling set-up for an ion-beam sputtering. As described in the text, an ion beam trajectory makes an angle of $\theta$ with the axis $z$, and the projection of the ion-beam direction on the $x-y$ plane makes an angle of $\phi_{\text {exp }}$ relative to the $x$ axis. Anisotropic direction is perpendicular to the $x-y$ projection of the ion-beam.

distribution. ${ }^{52}$ The share of energy for each lateral atom of the solid is calculated based on the Gaussian distribution, and each lateral atom is eroded with a probability proportional to that energy. In each diffusion sweep, hops to nearest neighbor sites are checked for all atoms with empty neighbors. Here, the probability of acceptance of a possible hop is calculated based on an Arrhenius hopping rate of the form of $P=k_{0} \exp \left(-\Delta E / k_{\mathrm{B}} T\right)$, where $k_{0}$ is a temperature dependent and material specific attempt rate, $\Delta E$ is an energy barrier assigned to the different possible local configurations of the lattice before and after a hop, $k_{\mathrm{B}}$ is the Boltzmann constant, and $T$ is the surface temperature.

Upon varying values of parameters and irradiation time length, different kinds of isotropic and anisotropic surface profiles can be produced. ${ }^{27}$ Here, we fix all the parameters except $\theta$ and $\phi_{\text {exp }}$. Examples of surface profile in size of $L=256$ after shooting 30 (atom/lateral atom) at different beam directions are depicted in Fig. 2.

\section{METHODOLOGY: CROSSING STATISTICS ANALYSIS}

After the initial introduction by Rice, ${ }^{53}$ the level crossing statistics has been improved and used to investigate up-crossing and down-crossing of a given stochastic field. During the last decades, many researches have examined its capabilities in studying growing processes in $1 \mathrm{D}, 2 \mathrm{D}$, and 3D. ${ }^{54-61}$ In this study, we use mentioned method to characterize isotropic and anisotropic rough surfaces. Also the curve-crossing method is another useful method for this purpose. $^{62}$

As explained in Sec. I, we are seeking a criterion to distinguish isotropic and anisotropic rough surfaces, to that goal, the crossing statistics method will be carried out in a $2 \mathrm{D}$ framework. Some advantages of this approach are as follows: in the common methods, there is no possibility to analyze the dependence of anisotropy on the threshold parameter, whereas in the crossing statistics, the threshold parameter can be systematically changed and the anisotropy can be recalculated. In addition, crossing statistics is robust against more complicated correlation functions as well as various forms of probability density functions of the underlying fluctuation. In other
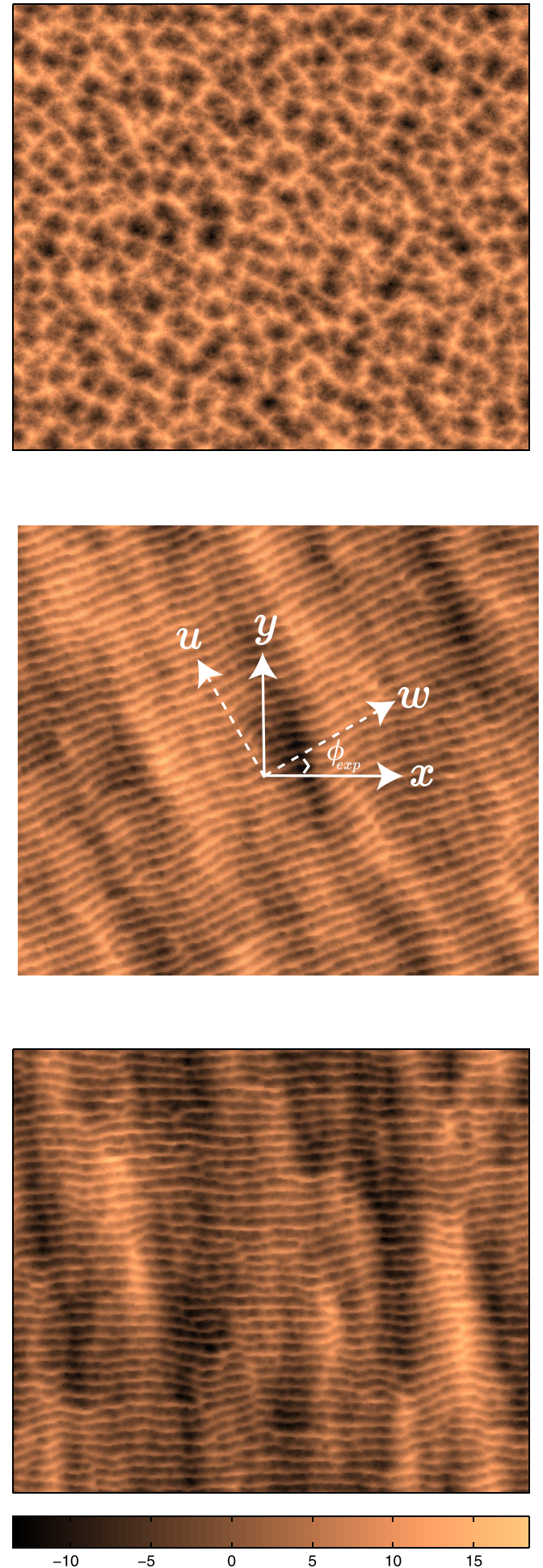

FIG. 2. Upper: Isotropic simulated rough surface for $\theta=0^{\circ}$ and $\phi_{\exp }=0^{\circ}$. Middle: A preferred direction for $\theta=25^{\circ}$ and $\phi_{\exp }=23^{\circ}$ exists for simulated surface. Lower panel corresponds to simulated anisotropic rough surface for $\theta=50^{\circ}$ and $\phi_{\exp }=0^{\circ}$. The color-bar is in an arbitrary unit.

words, the non-Gaussianity of the underlying rough surface can be handled by this method simultaneously. In the presence of isotropy and homogeneity, the crossing statistics for 2D and 3D stochastic fields can be expressed in terms of the statistics of 1D slices of the surface. ${ }^{54-61}$

Crossing statistics has been used to examine cosmological stochastic fields for example and its features have been 
investigated in different domains. ${ }^{54-57}$ In the following, we describe the sequential steps in analyzing the crossing statistics of a surface:

Step1: Definition of variables: Suppose that for a $2 \mathrm{D}$ rough surface, height fluctuations are represented by $\mathcal{H}(\mathbf{r})$ at coordinate $\mathbf{r}=(n, m)$ with resolution $\Delta$ and size $L \times L$ (see Fig. 3). For convenience, suppose that the origin of the coordinate system is located at the center of the rough surface. We describe the height fluctuations by $\mathcal{H}\left(x_{n}, y_{m}\right)$, where $x_{n}$ and $y_{m}$ represent the coordinate position. Crossing points with a positive slope at an arbitrary threshold, $\vartheta=\alpha / \sigma_{0}$, are called up-crossings (indicated by $\times$-symbols in the upper panel of Fig. 4). Here $\alpha$ and $\sigma_{0}$ are the values of the surface height and the variance of the height fluctuations, respectively. The extension of crossing statistics for a $2 \mathrm{D}$ rough surface requires considering iso-height contours at a given threshold, while for a 3D stochastic field, crossing statistics is achieved by considering iso-density surfaces. ${ }^{54}$

Step 2: Preparing data sets: We consider two categories of 1D slices of the height profile in two separate orthogonal directions labeled by $u$ and $w$ (Fig. 2). These two directions could be produced by a counterclockwise rotation of an angle $\phi$ (middle panel of Fig. 2). We indicate the variation of the surface along these two directions by $\mathcal{H}_{u}(\phi ; n, m)$ and $\mathcal{H}_{w}(\phi ; n, m)$. The size of these 1D slices depends on the resolution and the direction of slicing of the underlying rough surface. The upper panel of Fig. 4 shows a schematic illustration of height fluctuations along a given direction. If $\mathcal{H}(\mathbf{r})$ is invariant under Eulerian rotation, the statistical isotropy is present. For an isotropic and homogeneous profile, in long run, the up-crossings and down-crossings are statistically equivalent. ${ }^{61}$ Throughout this paper, we focus on upcrossings.

Step 3: Theoretical calculations: Probability distribution function (PDF) of the height of a rough surface is represented by $\mathcal{P}(\mathcal{H})$ and the corresponding conditional PDF is defined by $\mathcal{P}_{\eta}(\vec{\eta} \mid \mathcal{H})$, here $\vec{\eta} \equiv \vec{\nabla} \mathcal{H}$. The gradient of the height can be written as: $\vec{\eta}=\eta_{u} \hat{u}+\eta_{w} \hat{w}$. As discussed

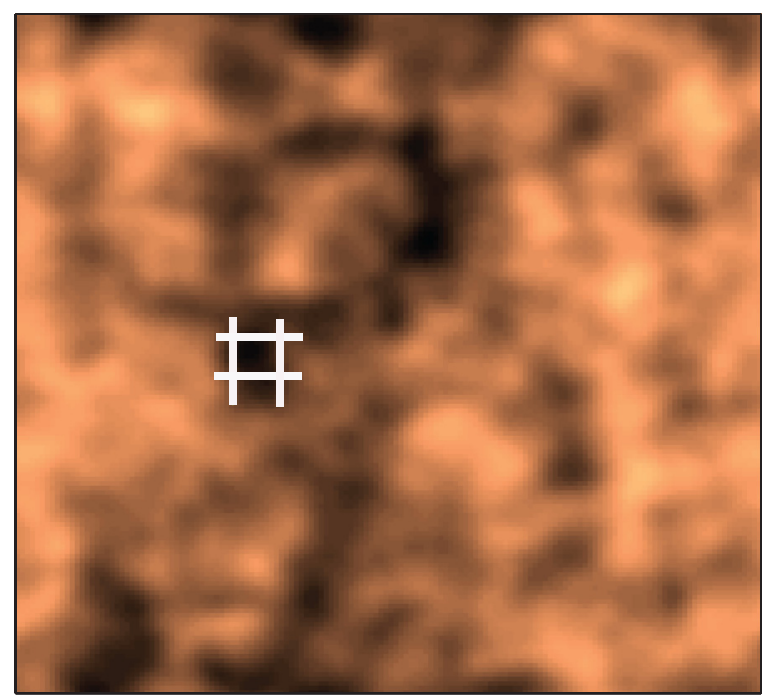

FIG. 3. Typical surface with a cell represented by a square. The size of mesh equates to resolution of underlying rough surface.

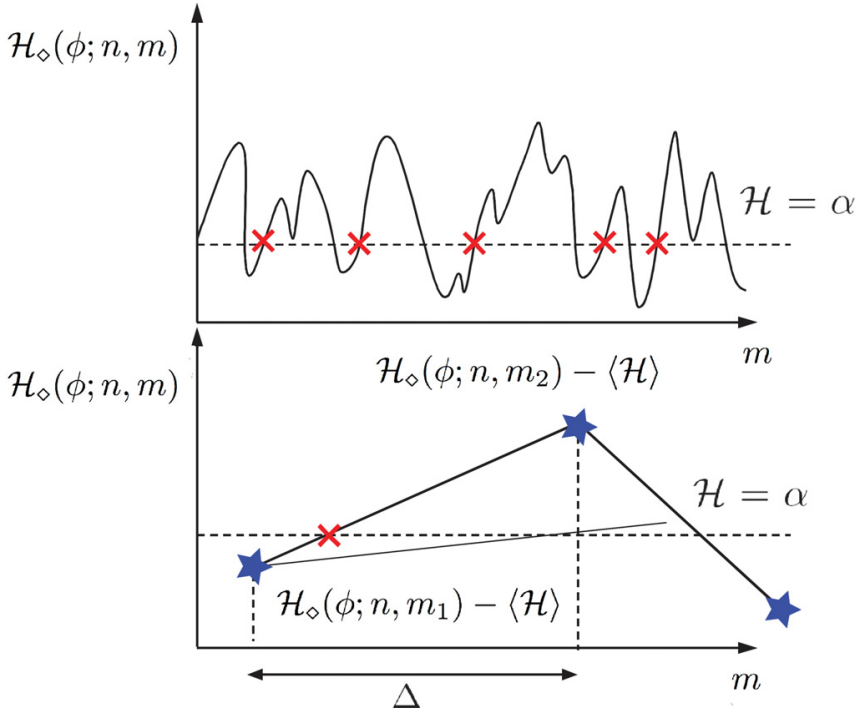

FIG. 4. Upper panel corresponds to a typical 1D surface with its positive slope crossing at the level $\mathcal{H}=\alpha$ marked by $\times$-symbols. Lower panel shows the necessary and sufficient conditions to have up-cross at threshold $\mathcal{H}=\alpha$.

before, for both $u$ and $w$ directions, we construct one dimensional slices of the height fluctuations as $\mathcal{H}_{\diamond}(\phi ; n, m)$, in which $\diamond$ symbol is replaced by $u$ and $w . n$ and $m$ run from 1 to $N$ and the sample size is $L=\Delta \times N$. We define $n_{\diamond}^{+}(\phi ; \alpha)$ as the number of up-crossings of the height profile at a given level $\alpha$ (see Fig. 2 for more details). For convenience, we set $\alpha \equiv \mathcal{H}_{\diamond}(\phi ; n, m)-\langle\mathcal{H}\rangle$. The ensemble average for level crossing with a positive slope is given by

$$
N_{\diamond}^{+}(\phi ; \alpha, L)=\left\langle n_{\diamond}^{+}(\phi ; \alpha, L)\right\rangle .
$$

In order to have up-crossing condition at level $\alpha$, the following two necessary and sufficient conditions should be satisfied (see the lower panel of Fig. 4)

(I) $\mathcal{H}_{\diamond}\left(\phi ; n, m_{1}\right)-\langle\mathcal{H}\rangle \leq \alpha$ and

(II) the slope of $\mathcal{H}_{\diamond}(\phi ; n, m)$ to be larger than or equal to the slope of a line connecting the starting point of the interval and the level $\alpha$ :

$$
\eta_{\diamond}\left(\phi ; n, m_{1}\right) \geq \frac{\alpha-\left[\mathcal{H}_{\diamond}\left(\phi ; n, m_{1}\right)-\langle\mathcal{H}\rangle\right]}{\Delta} .
$$

Considering the joint PDF of the height fluctuations and its derivative, $\mathcal{P}(\vec{\eta}, \mathcal{H})$, the region corresponding to $\mathbf{I}$ $\left(\mathcal{H}_{\diamond} \leq \alpha\right)$ and II $\left(\eta_{\diamond} \geq\left(\alpha-\mathcal{H}_{\diamond}\right) / \Delta\right)$ conditions in the plane $\left(\mathcal{H}_{\diamond}(\phi ; n), \eta_{\diamond}\right)$ is associated with the probability of having upcrossing in the direction $\diamond$ at level $\alpha$. In Fig. 5, the shaded volume corresponds to probability of having crossing with a positive slope at a given threshold, $\mathcal{H}_{\diamond}=\alpha \cdot{ }^{60}$ Subsequently, the probability of having up-crossing in the interval $\Delta$ is given by

$$
\begin{aligned}
N_{\diamond}^{+}(\phi ; \alpha, \Delta) & =\Delta \times \nu_{\diamond}^{+}(\phi ; \alpha) \\
& =\int d \vec{\eta} \quad \Theta\left(\eta_{\diamond}\right) \int_{\alpha-\left|\eta_{\diamond}\right| \Delta}^{\alpha} \mathcal{P}\left(\phi ; \vec{\eta}, \mathcal{H}_{\diamond}\right) d \mathcal{H}_{\diamond}
\end{aligned}
$$

in which $\Theta(:)$ is the step function. Therefore 


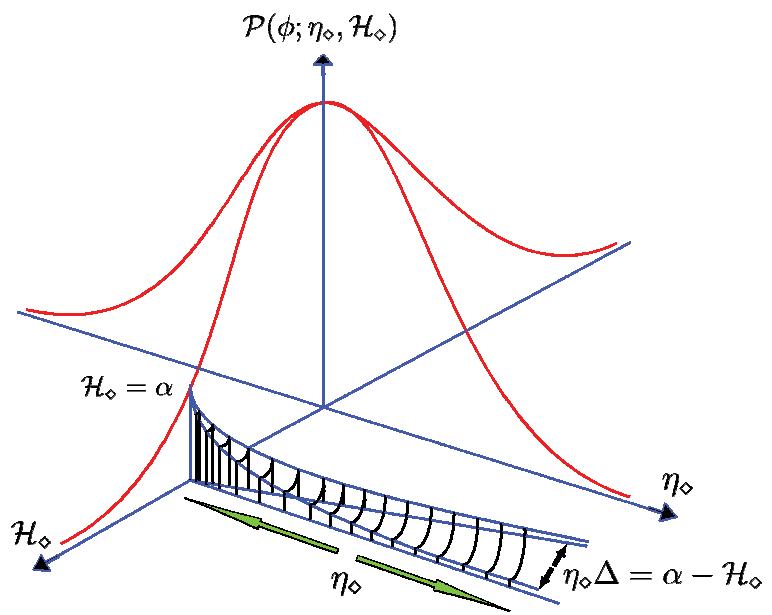

FIG. 5. Sketch of joint probability density function of a typical surface and its derivative with respect to corresponding dynamical parameter (position) in the level crossing theory. The shaded area corresponds to the total probability of finding crossings with a positive slope at the level $\mathcal{H}_{\diamond}=\alpha$. The $\diamond$ symbols can be replaced for each direction. The original idea of this plot has been given in Ref. 60.

$$
\begin{aligned}
\nu_{\diamond}^{+}(\phi ; \alpha) & =\int_{0}^{\infty} d \eta_{\diamond}\left|\eta_{\diamond}\right| \quad \overline{\mathcal{P}}\left(\phi ; \eta_{\diamond}, \mathcal{H}_{\diamond}=\alpha\right) \\
& =\mathcal{P}(\phi ; \alpha) \int_{0}^{\infty} d \eta_{\diamond}\left|\eta_{\diamond}\right| \quad \overline{\mathcal{P}}_{\vec{\eta}}\left(\phi ; \eta_{\diamond} \mid \alpha\right)
\end{aligned}
$$

where $\overline{\mathcal{P}}\left(\phi ; \eta_{\diamond}, \mathcal{H}_{\diamond}=\alpha\right)$ has been marginalized over other component of $\vec{\eta}$ vector (hereafter we remove bar symbol for convenience). $\nu_{\diamond}^{+}(\phi ; \alpha)$ is the number of up-crossings at level $\mathcal{H}_{\diamond}(\phi ; n, m)-\langle\mathcal{H}\rangle=\alpha$. In other words, $\nu_{\diamond}^{+}(\phi ; \alpha)^{-1}$ corresponds to wavelength of having an up-crossing event at level $\alpha$ through the direction $\diamond$. The most familiar form of Eq. (5) is $\nu_{\diamond}^{+}(\phi ; \alpha)=\mathcal{P}(\phi ; \alpha)\left\langle\left|\eta_{\diamond}\right| \Theta\left(\eta_{\diamond}\right)\right\rangle_{\alpha}$. In addition, if $\mathcal{P}_{\vec{\eta}}(\phi$; $\vec{\eta} \mid \alpha)=\mathcal{P}_{\vec{\eta}}(\phi ; \vec{\eta})$ which is the case for a homogeneous and isotropic Gaussian field, then Eq. (5) becomes $\nu_{\diamond}^{+}(\phi ; \alpha)$ $\sim \mathcal{P}\left(\phi ; \mathcal{H}_{\diamond}=\alpha\right)$. From the theoretical point of view, one can calculate up-crossing statistic using the functional form of joint PDF of the relevant variables. In the case of multivariate Gaussian joint PDF, we have

$$
\mathcal{P}(\mathbf{A})=\sqrt{\frac{\operatorname{det} \mathcal{M}}{(2 \pi)^{3}}} e^{-\frac{1}{2}\left(\mathbf{A}^{T} \cdot \mathcal{M} \cdot \mathbf{A}\right)},
$$

where $\mathbf{A} \equiv\left\{\mathcal{H}, \eta_{u}, \eta_{w}\right\}$ and $\mathcal{M}$ is the inverse of the covariance matrix of the underlying variables

$$
\mathcal{M}^{-1} \equiv \operatorname{Cov}=\left[\begin{array}{ccc}
\left\langle\mathcal{H}^{2}\right\rangle & \left\langle\mathcal{H} \eta_{w}\right\rangle & \left\langle\mathcal{H} \eta_{u}\right\rangle \\
\left\langle\eta_{w} \mathcal{H}\right\rangle & \left\langle\eta_{w}^{2}\right\rangle & \left\langle\eta_{w} \eta_{u}\right\rangle \\
\left\langle\eta_{u} \mathcal{H}\right\rangle & \left\langle\eta_{u} \eta_{w}\right\rangle & \left\langle\eta_{u}^{2}\right\rangle
\end{array}\right]
$$

Each element of covariance matrix can be computed using the power spectrum of the underlying 2D rough surface, $S^{(2 \mathrm{D})}(\mathbf{k})$. We derived these elements for an m-dimensional isotropic stochastic field in the Appendix. We suppose that $\langle\mathcal{H}\rangle=0$; therefore, the analytical form of up-crossing statistics for isotropic rough surface for arbitrary slice [Eq. (5)] becomes

$$
\begin{aligned}
\nu_{\diamond}^{+}(\alpha) & =\mathcal{P}(\alpha)\left\langle\left|\eta_{\diamond}\right| \Theta\left(\eta_{\diamond}\right)\right\rangle_{\alpha} \\
& =\frac{1}{2 \pi \sqrt{2}} \frac{\sigma_{1}}{\sigma_{0}} \mathbf{e}^{-\alpha^{2} / 2 \sigma_{0}^{2}},
\end{aligned}
$$

where $\sigma_{0}$ and $\sigma_{1}$ are spectral parameters (see the Appendix for the definition).

In a general case, the up-crossing reads as

$$
\nu_{\diamond}^{+}(\alpha)=\left\langle\delta_{d}(\mathcal{H}(\mathbf{r})-\alpha)\left|\eta_{\diamond}\right| \Theta\left(\eta_{\diamond}\right)\right\rangle .
$$

Here, $\delta_{d}$ is the Dirac delta function. In addition to the abovementioned definition of up-crossing, the conditional upcrossing introduced in Ref. 55 is

$$
\nu_{x}^{+}(\alpha \mid \text { cond. })=\left\langle\delta_{d}(\mathcal{H}(\mathbf{r})-\alpha)\left|\eta_{x}\right| \Theta\left(\eta_{x}\right) \delta_{d}\left(\eta_{y}\right)\left|\xi_{y y}\right|\right\rangle .
$$

The perturbation formula for Eq. (9) up to $\mathcal{O}\left(\sigma_{0}^{2}\right)$ is given in Ref. 56, and for an isotropic Gaussian field in 2D, the closed form of Eq. (10) is worked out in Ref. 55. As we are going to use this method for probing anisotropies imposed on a typical rough surface in $2 \mathrm{D}$, we can express that the up-crossing in an arbitrary direction based on the 1D power spectrum, $S^{(1 \mathrm{D})}(k)$, as in Ref. 56

$$
\nu_{\diamond}^{+}(\alpha ; 1 \mathrm{D})=\frac{1}{2 \pi} \frac{\sigma_{1}(1 \mathrm{D})}{\sigma_{0}} \mathbf{e}^{-\alpha^{2} / 2 \sigma_{0}^{2}},
$$

where

$$
\sigma_{1 \diamond}^{2}(1 \mathrm{D})=\frac{L}{2 \pi} \int d k_{\diamond} k_{\diamond}^{2} S^{(1 \mathrm{D})}\left(k_{\diamond}\right)
$$

and the $1 \mathrm{D}$ power spectrum is given by

$$
S^{(1 \mathrm{D})}\left(k_{1}\right)=\frac{L}{2 \pi} \int d k_{2} S^{(2 \mathrm{D})}(\mathbf{k})
$$

For an isotropic rough surface, $\sigma_{1}^{2}(2 \mathrm{D})=2 \sigma_{1 \diamond}^{2}(1 \mathrm{D})$, and consequently $\nu_{\diamond}^{+}(\alpha ; 1 \mathrm{D})=\nu_{\diamond}^{+}(\alpha)$.

For a Gaussian anisotropic rough surface, we use the power spectrum given in Eq. (1) belonging to the correlation length anisotropic model. The up-crossing in the direction $\diamond$ is

$$
\nu_{\diamond}^{+}(\alpha)=\frac{1}{2 \pi \sqrt{2(\gamma-1)}} \frac{k_{c}}{\xi_{\diamond}} \mathbf{e}^{-\alpha^{2} / 2 \sigma_{0}^{2}} .
$$

Therefore for an anisotropic Gaussian rough surface, the ratio of up-crossing in two anisotropic directions is $\nu_{u}^{+}(\alpha) / \nu_{w}^{+}(\alpha)=\xi_{w} / \xi_{u}$ representing the inverse ratio of corresponding correlation length scales.

For a scaling anisotropic model, the power spectrum introduced in Eq. (2) is implemented. Therefore, up-crossing in the direction $\diamond$ becomes

$$
\nu_{\diamond}^{+}(\alpha)=\frac{1}{2 \pi \sqrt{2\left(\gamma_{\diamond}-1\right)}} \frac{k_{c}}{\xi_{\diamond}} \mathbf{e}^{-\alpha^{2} / 2 \sigma_{0}^{2}},
$$

and in this case we have

$$
\frac{\nu_{u}^{+}(\alpha)}{\nu_{w}^{+}(\alpha)}=\sqrt{\frac{\gamma_{w}-1}{\gamma_{u}-1}} \frac{\xi_{w}}{\xi_{u}} .
$$


Another useful parameter based on $\nu_{\diamond}^{+}(\phi ; \alpha)$ is the generalized up-crossing defined as

$$
N_{\text {tot }}^{\diamond}(\phi ; q) \equiv \int_{-\infty}^{+\infty} \nu_{\diamond}^{+}(\phi ; \alpha)|\alpha-\langle\mathcal{H}\rangle|^{q} d \alpha
$$

Clearly, if $q=0, N_{t o t}^{\diamond}(\phi ; q)$ specifies the total number of up-crossings for the height fluctuations with a positive slope at all levels in the direction $\diamond$. For a typical rough surface, $N_{\text {tot }}^{\diamond}(\phi, q=0)$ can be considered as a measure of roughness. For a typical long-range correlated surface, $N_{\text {tot }}^{\diamond}(\phi, q=0)$ is smaller than that of a shuffled surface, while for an anticorrelated surface $N_{\text {tot }}^{\diamond}(\phi, q=0)$ has to be larger than that of a completely un-correlated process. For an isotropic Gaussian rough surface, we have

$$
N_{t o t}^{\diamond}(q)=\frac{\sigma_{1}}{\sigma_{0}} \frac{2^{\frac{q}{2}-1}}{\pi} \Gamma\left(\frac{1+q}{2}\right), \quad q>-1 .
$$

For a correlated anisotropic Gaussian surface, by using Eqs. (1) and (13), Eq. (16) reads as

$$
N_{t o t}^{\diamond}(q)=\frac{k_{c} 2^{\frac{q}{2}-1}}{\pi \sqrt{\gamma-1} \xi_{\diamond}} \Gamma\left(\frac{1+q}{2}\right), \quad q>-1
$$

while for the scaling exponent of the anisotropic Gaussian surface, we consider a power spectrum according to Eqs. (2) and (14); therefore, Eq. (16) becomes

$$
N_{\text {tot }}^{\diamond}(q)=\frac{k_{c} 2^{\frac{q}{2}-1}}{\pi \sqrt{\gamma_{\diamond}-1} \xi_{\diamond}} \Gamma\left(\frac{1+q}{2}\right), \quad q>-1 .
$$

The upper panel of Fig. 6 shows the isotropic Gaussian rough surface. The filled circle symbols in the middle panel of this figure correspond to the numerical computation of $\nu_{\diamond}^{+}(\alpha)$, while the solid line is calculated by Eq. (8), which is the theoretical prediction for the up-crossing as a function of $\alpha$. The generalized up-crossing statistics, $N_{\text {tot }}^{\diamond}(q)$, is shown in the lower panel. Our results demonstrate a good consistency between the numerical and theoretical predictions.

In Sec. IV, we are going to compute $\nu_{\diamond}^{+}(\phi, \alpha)$ for the height fluctuations in two distinct directions and then we try to find a robust criterion to distinguish isotropic and anisotropic surfaces.

\section{IMPLEMENTATION OF CROSSING STATISTICS ON ANISOTROPIC SURFACES}

After generating a 2D stochastic field via synthetic methods or preparing a rough surface in an experiment, an important question is whether a preferred direction has been imposed on the underlying stochastic field. Suppose there is an arbitrary feature on a given rough surface $\mathcal{H}(\mathbf{r})$. Statistical isotropy causes $\mathcal{H}(\mathbf{r})$ to be invariant under Eulerian transformation

$$
\langle\mathcal{H}(\mathbf{r})\rangle=\langle\mathcal{H}(\mathcal{R} \mathbf{r})\rangle
$$

here $\mathcal{R}$ represents the rotation matrix.

In order to quantify the probable anisotropy of the rough surface, we apply the up-crossing statistics method to
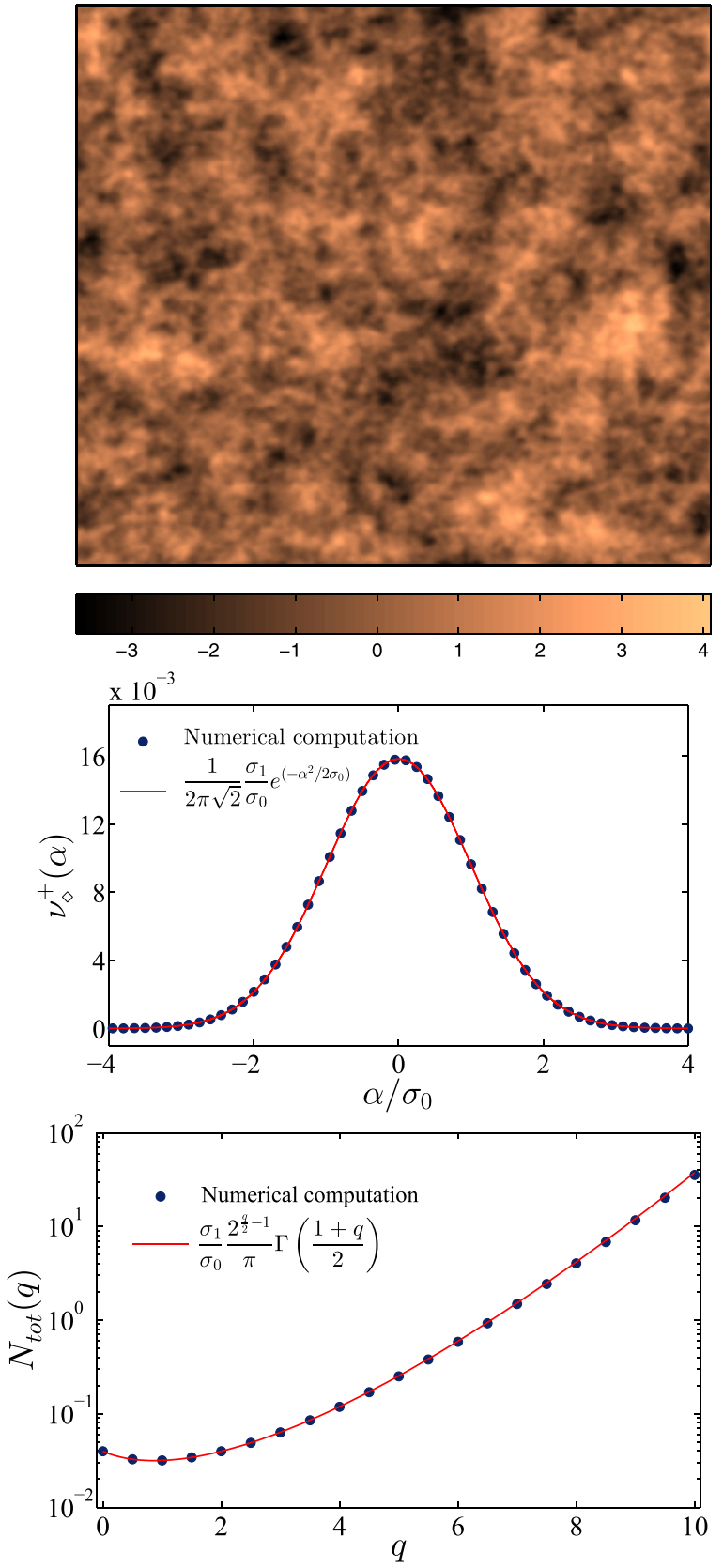

FIG. 6. Upper panel: Simulated isotropic Gaussian rough surface. Middle panel: Up-crossing analysis for the isotropic Gaussian rough surface. Lower panel is $N_{t o t}^{\diamond}(q)$ for the isotropic Gaussian rough surface. The red solid line represents the theoretical prediction and filled circles correspond to the numerical computation. The color-bar is in the unit of height fluctuation variance. The symbol size is equal to the statistical errors at $68 \%$ level of confidence.

calculate $\nu_{\diamond}^{+}$and $N_{\text {tot }}^{\diamond}$ of the surface. We expect that the upcrossing statistics for various directions on an isotropic rough surface to be statistically identical, while in an anisotropic case, $\nu^{+}(\alpha)$ takes different values at least for $\vartheta \equiv \alpha / \sigma_{0}=0$ in different directions. The upper panel of Fig. 7 confirms this. The lower panel corresponds to the same quantity for a synthetic anisotropic rough surface. In this plot, we select $u$ and $w$ axes to be aligned with the anisotropy direction imposed on the synthetic rough surface. In Fig. 8, we use the power spectrum for the correlation anisotropy [Eq. (1)]. Then we compute the crossing statistics for directions 

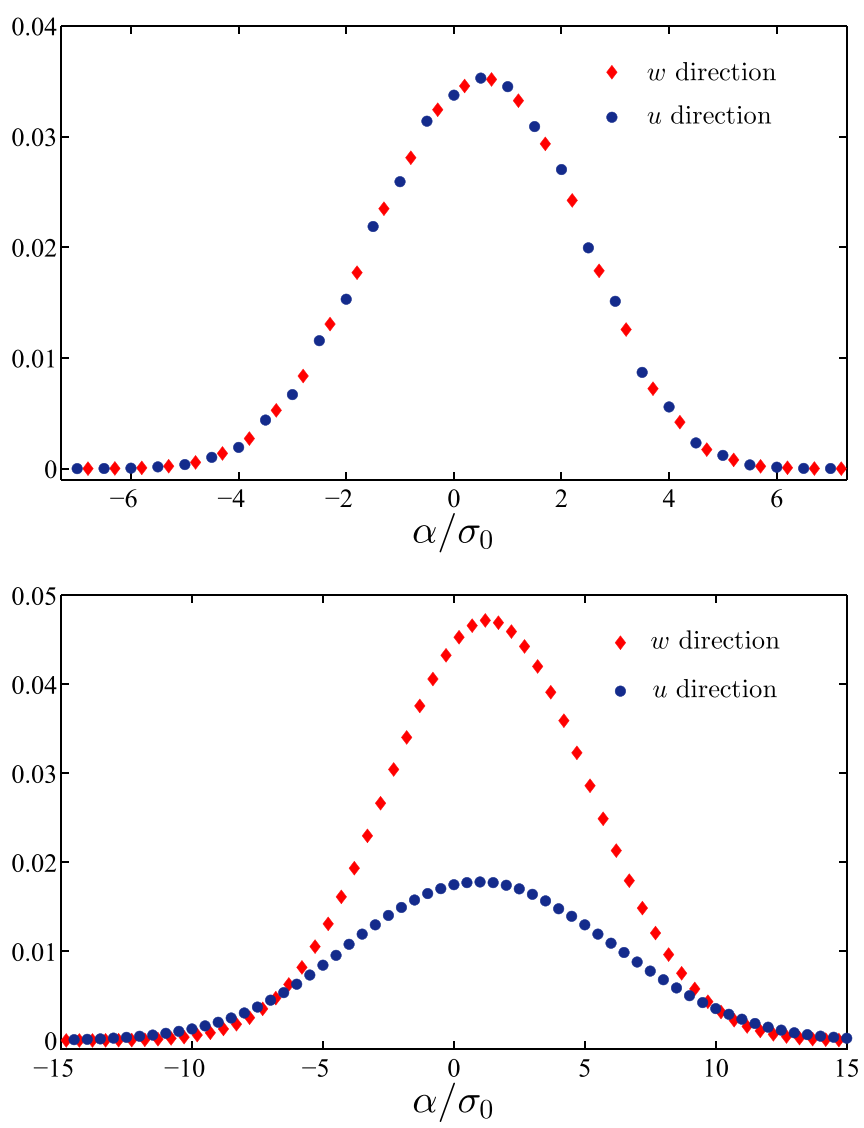

FIG. 7. Upper panel shows the up-crossing analysis for completely isotropic rough surface for two arbitrary directions. Lower panel corresponds to $\nu_{\alpha}^{+}$as a function of level for anisotropic rough surface through mentioned anisotropic directions. The symbol size is equal to the statistical errors at $1 \sigma$ confidence level.

parallel and perpendicular to the given anisotropic direction. The solid lines in the middle and lower panels indicate the theoretical prediction. Fig. 9 contains same information except for the scaling exponent anisotropy.

To compare our method with other approaches, for example, the power spectral density, we compute the power spectrum for scaling anisotropic simulations. Figure 10 demonstrates the scaling behavior of the anisotropic surface. The scaling exponents of the power spectrum are compatible with those used for the simulations. Worth to mention is that for $k_{c} \rightarrow \infty$ corresponding to $r_{c} \rightarrow 0$, the precision of the computed scaling spectra decreases due to the finite size effects, while the crossing statistics is robust due to the fact that the corresponding analysis is performed in the real space.

To utilize the power of the crossing statistics in detecting the direction of the anisotropy, we use an anstz as

$$
\mathcal{Q}^{2}(\phi, q) \equiv \sum_{n=1}^{\mathcal{N}} \frac{\left[N_{t o t}^{w}(n ; \phi, q)-N_{t o t}^{u}(n ; \phi, q)\right]^{2}}{\left[\sigma_{w}^{2}(n ; \phi, q)+\sigma_{u}^{2}(n ; \phi, q)\right]} .
$$

Here, $\sigma_{\diamond}(n ; \phi, q)$ denotes the error bar of the generalized upcrossing and $n$ runs from 1 up to the total number of sample profiles. Since we are looking for the magnitude of rotation $(\phi)$, for which the difference in the generalized up-crossing is maximum, we measure $\mathcal{Q}^{2}(\phi, q)$ as a function of $\phi$
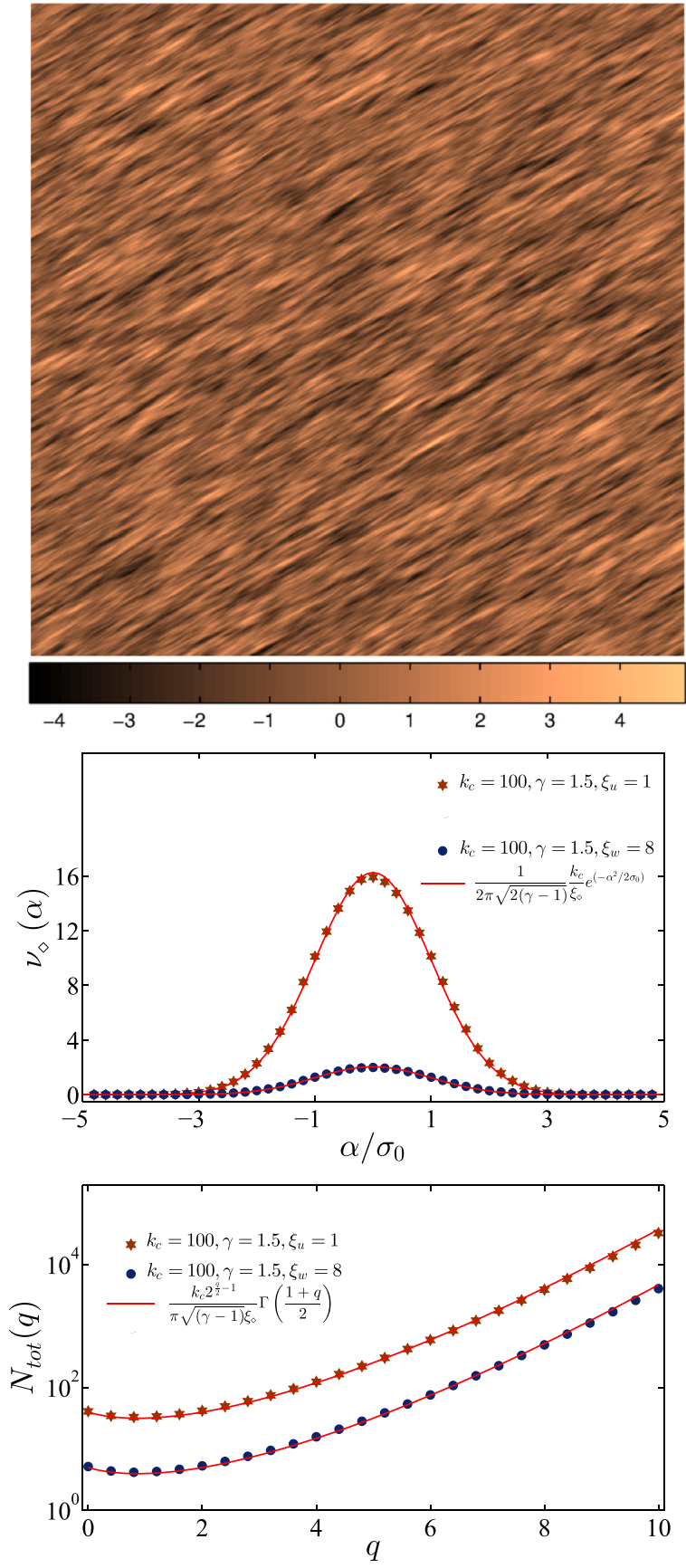

FIG. 8. Upper panel: Simulated anisotropic Gaussian rough surface with the power spectrum given by Eq. (1). Middle panel: Up-crossing analysis for the correlation length anisotropic Gaussian rough surface. Lower panel is $N_{\text {tot }}^{\diamond}(q)$ for the same simulated rough surface. The red solid line represents the theoretical prediction and the filled circles correspond to the numerical computation. The color-bar is in the unit of the height fluctuation variance. The symbol size is proportional to the statistical errors at $1 \sigma$ confidence level.

for each value of $q$ and, by estimating the P-value for this quantity, quantify the degree of reliability. Fig. 11 shows $\mathcal{Q}^{2}(\phi)$ for $q=0$ as a function of $\phi$ for the synthetic anisotropic rough surface simulated by the IBS method with $\phi=23^{\circ}$. It demonstrates a peak in $\mathcal{Q}^{2}(\phi)$ around $\phi \sim 23^{\circ}$.

In order to quantify the degree of anisotropy in the underlying rough surface, we need to investigate the statistical deviation between $N_{t o t}^{u}(\phi, q)$ and $N_{t o t}^{w}(\phi, q)$. The significance of this deviation is systematically checked by 

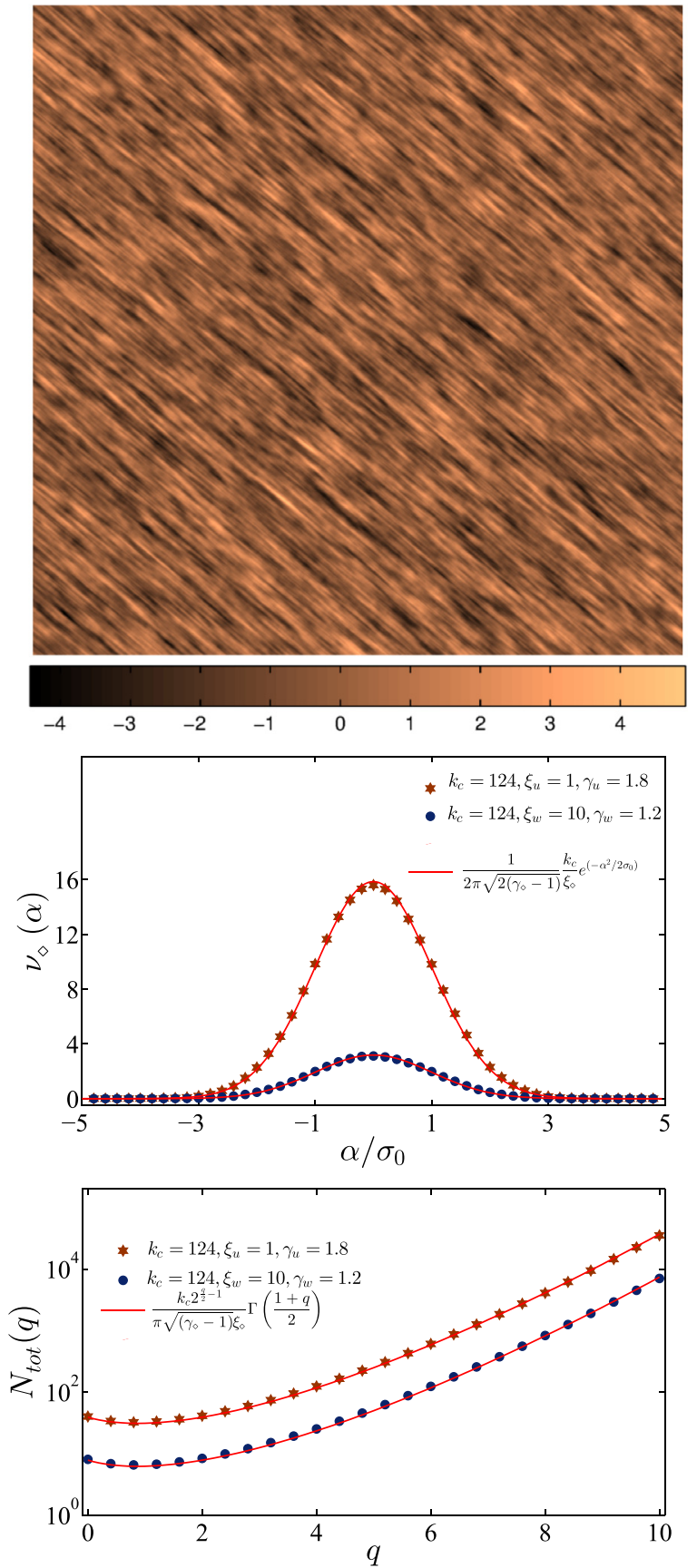

FIG. 9. Upper panel: Synthetic anisotropic Gaussian rough surface with correlation and scaling exponent anisotropies [Eq. (2)]. Middle panel: Upcrossing analysis of the anisotropic Gaussian rough surface. Lower panel is $N_{t o t}^{\diamond}(q)$ for the same simulated rough surface. The red solid line represents the theoretical prediction and the filled circles correspond to the numerical computation. The color-bar is in the unit of the height fluctuation variance. The symbol size is proportional to the statistical errors at $1 \sigma$ confidence level.

calculating Student's $t$-test for equal sample sizes and unequal means and variances for each $q$ and $\phi$ according to

$$
\begin{aligned}
t(\phi, q)= & {\left[N_{\text {tot }}^{u}(\phi, q)-N_{\text {tot }}^{w}(\phi, q)\right] } \\
& \times \sqrt{\frac{N_{\text {run }}}{\sigma_{u}^{2}(\phi, q)+\sigma_{w}^{2}(\phi, q)}},
\end{aligned}
$$

where $N_{\text {run }}$ indicates the index of the ensemble which is equal to $n$ introduced in Sec. III. The P-value, corresponding

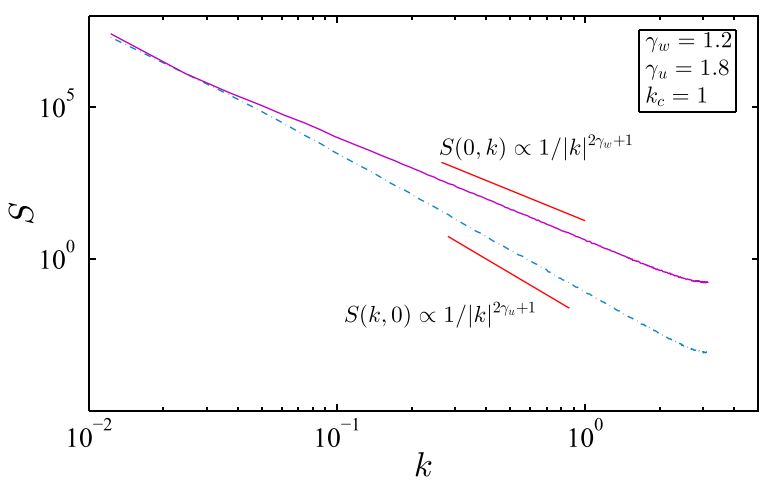

FIG. 10. This figure demonstrates that scaling exponents of the scaling exponents' anisotropic model are in agreement with those we used for the simulation. The solid line corresponds to the directional power spectrum for the $x$-direction and the dashed line is for the 1D power spectrum for the $y$-direction.

to $t(\phi, q)$ for $m=2 N_{\text {run }}-2$ degrees of freedom, is determined by two-tailed hypothesis: $\quad p(\phi, q)=2 \int_{t(\phi, q)}^{\infty}$ $\frac{\Gamma((m+1) / 2)}{\Gamma(m / 2)} \frac{1}{\sqrt{m \pi}}\left(1+\frac{x^{2}}{m}\right)^{-(m+1) / 2} d x$. The chi-square for the Pvalue reads as:

$$
\chi^{2}(\phi)=-2 \sum_{q=q_{\min }}^{q_{\max }} \ln p(\phi, q) .
$$

Finally, by using the chi-square distribution function for final P-value, $P_{\text {final }}(\phi)$, associated with $\chi^{2}(\phi)$ and with $\mu \equiv 2\left(\frac{q_{\max }-q_{\min }}{\Delta q}\right)-2$ degrees of freedom, is computed as

$$
P_{\text {final }}(\phi)=1-\frac{1}{2^{\mu / 2} \Gamma(\mu / 2)} \int_{0}^{x^{2}(\phi)} e^{-x / 2} x^{\mu / 2-1} d x .
$$

For $3 \sigma$ significance level, $P_{\text {final }}(\phi)<0.0027$, we can conservatively say that there exists a significant difference between the two generalized up-crossing quantities for the two directions, $u$ and $w$ at the given $\phi$. Fig. 12 represents the P-value for the anisotropic rough surface shown in Fig. 11.

The crossing statistics also enables us to investigate the type of anisotropy of the rough surface. Correlation length anisotropy and scaling exponent anisotropies are ubiquitous in simulations and experiments. In practice, to discriminate between these two types of anisotropies, one should compute Eq. (24). After determining the direction of anisotropy, the left hand side of Eq. (15) and/or Eq. (19) can be set based on the ratio $\nu_{u}^{+}(\alpha) / \nu_{w}^{+}(\alpha)$.

By the use of methods such as spectral analysis, ${ }^{63}$ fluctuation analysis, ${ }^{64}$ detrended fluctuation analysis (DFA), ${ }^{65-67}$ wavelet transform module maxima (WTMM) ${ }^{68-72}$ and discrete wavelets, ${ }^{73,74}$ the value of scaling exponents in $u$ and $w$ directions is determined, and finally by means of Eq. (15) and/or Eq. (19), the type of anisotropy and the ratio of the correlation length anisotropy are determined. It is worth noting that methods implemented for determining the scaling exponent usually give an accurate value for scaling exponent, while methods established for computing characteristic correlation length scale encounter the finite size effects. 

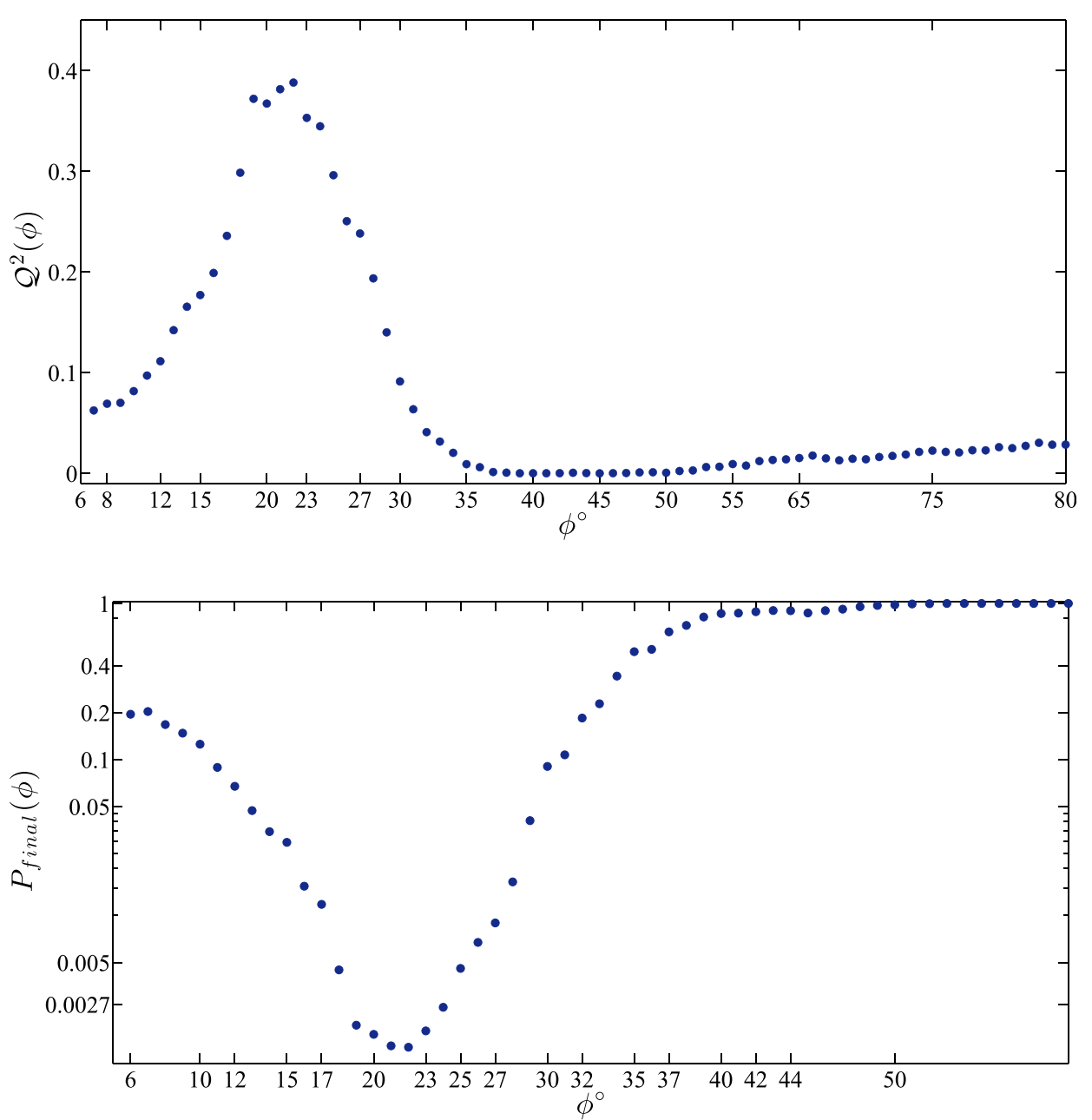

FIG. 11. The value of $\mathcal{Q}^{2}$ as a function of $\phi$ for the anisotropic rough surface illustrated in Fig. 2 with $\phi=23^{\circ}$.
The statistical periodicity of anisotropic patterns at a given threshold can be examined by the up-crossing statistics. As mentioned in Sec. III and can be found from Eq. (5), $\nu^{+}(\alpha)$ represents the wave-number at the threshold $\vartheta=\alpha / \sigma_{0}$; consequently, $1 / \nu^{+}(\alpha)$ shows the statistical characteristic length scale for the up-crossing at the threshold $\vartheta=\alpha / \sigma_{0}$. In addition, the generalized total number of crossing statistics [Eq. (16)] is a useful criterion to determine the type of the roughness for all thresholds, $\alpha$, in various directions. For example for $q=0$, Eqs. (16)-(18) represent the total roughness of the surface in a given direction.

\section{SUMMARY AND CONCLUSIONS}

Anisotropy and non-Gaussianity are two important properties of stochastic fields, which should be well addressed from the theoretical and experimental points of view. Several methods have been implemented to explore exotic features and properties of stochastic fields, but systematic limitations in theoretical and computational approaches cause discrepancies among these approaches. Based on the previous work on crossing statistics in various dimensions, ${ }^{53-61}$ in this paper we relied on the crossing statistics at a given threshold, $\vartheta \equiv \alpha / \sigma_{0}$, and introduced them as a robust benchmark for anisotropy detection imposed in stochastic fields in 2D. In addition, we showed that this method can examine the Gaussianity nature of $2 \mathrm{D}$ rough surfaces. According to an extensive study by Ryden, ${ }^{54}$ the crossing statistics for anisotropic field in $m \mathrm{D}$ is related to that of computed from one dimensional slices of underlying field. Subsequently, we used $\nu_{\diamond}^{+}(\alpha)$ for prepared slices parallel and perpendicular to a given direction, $\diamond$, and compared them to find the probable anisotropic direction. The generalized total crossing $N_{\text {tot }}^{\diamond}$ with a positive slope has been investigated for complementary tests. The characteristic wavelength or the characteristic length scale, $1 / \nu_{\diamond}^{+}$, at an arbitrary threshold, can be introduced in the context of the crossing statistics for further evaluation. From the theoretical point of view, considering the multivariate probability density function, we showed that the crossing statistics for an arbitrary slice in an isotropic Gaussian rough surface is given by Eq. (8) using a 2-dimensional power spectrum. We also derived perturbation expansions for upcrossing for $m \mathrm{D}$ isotropic stochastic fields. In addition, as introduced in Eq. (10), the theoretical prediction for upcrossing with applying additional conditions is straightforward to set up. ${ }^{55}$

In order to examine the direction of the anisotropy and to determine the type of the anisotropy in a $2 \mathrm{D}$ rough surface, we used two methods to synthesize isotropic and anisotropic rough surfaces. The first method is based on the modified Fourier filtering method with anisotropy imposed on the rough surface due to the correlation length scale [Eq. (1)] and/or due to the scaling anisotropic model [Eq. (2)]. We also used a Kinetic Monte Carlo (KMC) method to 
model the pattern formation by ion-beam sputtering (IBS). Up-crossing enumeration of the simulated isotropic Gaussian rough surface through different directions is in agreement with what is predicted by theoretical calculations (Fig. 6).

We assumed a set of orthogonal axes on the underlying 2D field labeled by $w$ (parallel) and $u$ (normal) with respect to the unknown anisotropic direction. Consequently, we determined $\nu_{\diamond}^{+}(\alpha)$ and $N_{\text {tot }}^{\diamond}(q)$ in both directions. The directional dependency of the difference between the computed results in those directions demonstrated that one can determine the imposed anisotropic direction.

In addition, specifying the type of the anisotropy in the rough surface is well motivated from the experimental point of view. Fig. 8 shows our results for a simulated correlation length anisotropic surface. Our results confirmed that the theoretical prediction for the ratio of the up-crossing statistics of $u$ and $w$ directions is compatible with that of determined by computation. Therefore, we are able to determine not only the direction of the anisotropy but also the ratio of the correlation length scales for $u$ and $w$ directions by using the quantity $\nu_{u}^{+} / \nu_{w}^{+}$.

For the anisotropy produced by different scaling exponents, we found consistent results reported in Fig. 9. To distinguish between the correlation length and scaling exponent anisotropies, according to Eq. (15), we should use prior information about the value of $\xi$ 's or $\gamma$ 's. Using a method to determine the scaling exponent, one can break this degeneracy and determine the type of the anisotropy and the ratio of the correlation lengths in $u$ and $w$ directions. It is worth noting that the methods for determining the scaling exponents are well established, whereas, because of the various definitions for the correlation length scale, computation of the characteristic scale is more challenging. However, the upcrossing analysis can determine the correlation length scale in a more robust approach.

The strategy for determining the direction of anisotropy is as follows: for both $w$ and $u$ directions on the anisotropic 2D surface, we computed $N_{\text {tot }}^{\diamond}(q)$ and the directional dependency of difference in generalized up-crossing by introducing $\mathcal{Q}^{2}(\phi, q)$ in Eq. (21). Subsequently, by computing the relevant P-value, we could recognize the anisotropic direction at the $3 \sigma$ confidence interval (Figs. 11 and 12).

Before finalizing this paper, some advantages of the upcrossing statistics as anisotropic probe are listed below:

(1) The crossing statistics is well established both theoretically and experimentally. We work with it in the real space and it is not affected by boundary effects. Also initial information is not modulated with the other auxiliary quantities in the phase space.

(2) It is possible to add an arbitrary condition for enumeration of the crossing statistics. It is also straightforward to set up a theoretical framework for this condition [Eq. (10)].

(3) From the computational point of view, one can apply this method to anisotropic non-Gaussian fields in arbitrary dimensions. In some cases, one can find an analytical solution for the statistics, such as the one presented in Ref. 55. This method is able to determine the
non-Gaussianity nature accompanying the anisotropy. The contribution of different scales in the detected anisotropy can be examined by varying the value of $q$.

(4) One can determine various characteristic length scales for an arbitrary threshold in the context of crossing statistics.

(5) In some cases, e.g., isotropic Gaussian stochastic field, the up-crossing statistics for higher dimensions can be written in terms of the crossing statistics in lower dimensions.

(6) The generalized up-crossing, $N^{+}(q)$, gives also a criterion for determining the roughness ${ }^{75}$ and other exotic features. ${ }^{59}$

We propose to use the described pipeline in examples of real stochastic fields in condensed matter, cosmology, and astrophysics.

\section{ACKNOWLEDGMENTS}

S.M.S.M. is grateful to Ravi K. Sheth, A. Vafaei Sadr, and S. Bazmi for their comments on the perturbation approach. S.M.S.M. and S.M.V.A. are grateful to the associate and federation office of ICTP for their support and the hospitality of CMSP and HECAP sections of ICTP, where some parts of this analysis were carried out. This research has been financially supported by Shahid Beheshti University research deputy affairs under annual grant and school of physics, IPM. The work of S.M.V.A. was supported in part by the Research Council of the University of Tehran.

\section{APPENDIX: PERTURBATIVE EXPANSIONS}

In this appendix, we will provide detailed derivations of some of the equations used in the paper.

For a stochastic field in $m \mathrm{D}$, we consider a covariant vector field containing relevant quantities of the underlying stochastic field as: $A_{\beta}:\left\{\alpha, \vec{\eta}, \xi_{i j}\right\}$, where $\alpha$ represents the value of the stochastic field $(\mathcal{H}(\mathbf{r})), \eta$ 's are the first derivative, and $\xi_{i j}$ 's correspond to the second derivative with respect to the independent parameter in $i$ and $j$ directions. The correlation function of the stochastic field becomes

$$
\begin{aligned}
C_{\mathcal{H}}(\mathbf{R}) & \equiv\langle\mathcal{H}(\mathbf{r}+\mathbf{R}) \mathcal{H}(\mathbf{r})\rangle \\
& =\frac{L^{m}}{(2 \pi)^{m}} \int d \mathbf{k} S^{(m \mathrm{D})}(\mathbf{k}) e^{\mathrm{ik} \cdot \mathbf{R}} .
\end{aligned}
$$

The spectral parameters are

$$
\begin{aligned}
\sigma_{0}^{2} \equiv\left\langle\mathcal{H}(\mathbf{r})^{2}\right\rangle=\frac{L^{m}}{(2 \pi)^{m}} \int d \mathbf{k} S^{(m \mathrm{D})}(\mathbf{k}) . \\
\sigma_{n}^{2} \equiv\left\langle\left(\frac{\partial^{n} \mathcal{H}(\mathbf{r})}{\partial x^{n}}\right)^{2}\right\rangle \\
=\frac{L^{m}}{(2 \pi)^{m}} \int d \mathbf{k} k^{2 n} S^{(m \mathrm{D})}(\mathbf{k}) .
\end{aligned}
$$

For the isotropic rough surface, we can write 


$$
\begin{aligned}
\left\langle\mathcal{H} \eta_{u_{j}}\right\rangle & =\left\langle\mathcal{H} \frac{\partial \mathcal{H}}{\partial u_{j}}\right\rangle \\
& =\frac{L^{m}}{(2 \pi)^{m}} \int d \mathbf{k i} k_{u_{j}} S^{(m \mathrm{D})}(\mathbf{k}) e^{\mathbf{i k} . \mathbf{R}}=0 .
\end{aligned}
$$

The correlation functions of the derivatives of the stochastic field in the isotropic case are

$$
\begin{aligned}
\left\langle\eta_{w}^{2}\right\rangle & =\frac{L^{m}}{(2 \pi)^{m}} \int d \mathbf{k} k_{w}^{2} S^{(m \mathrm{D})}(\mathbf{k}) \\
\left\langle\eta_{u}^{2}\right\rangle & =\frac{L^{m}}{(2 \pi)^{m}} \int d \mathbf{k} k_{u}^{2} S^{(m \mathrm{D})}(\mathbf{k}) \\
& =\frac{1}{m}\left\langle\eta^{2}\right\rangle=\frac{1}{m} \sigma_{1}^{2},
\end{aligned}
$$

where

$$
\left\langle\eta^{2}\right\rangle=\frac{L^{m}}{(2 \pi)^{m}} \int d \mathbf{k} k^{2} S^{(m \mathrm{D})}(\mathbf{k}) .
$$

Using the correlation function, we can write

$$
\begin{aligned}
\left\langle\mathcal{H} \frac{\partial^{2} \mathcal{H}}{\partial u_{i} \partial u_{j}}\right\rangle & =-\left\langle\frac{\partial \mathcal{H}}{\partial u_{i}} \frac{\partial \mathcal{H}}{\partial u_{j}}\right\rangle \\
& =-\frac{1}{m} \sigma_{1}^{2} \delta_{i j} .
\end{aligned}
$$

To compute the up-crossing statistics, we should also determine the statistical average of the absolute value of the derivative of the underlying stochastic field, so for a multivariate Gaussian PDF, we have

$$
\begin{aligned}
\left\langle\left|\eta_{u_{i}}\right|\right\rangle & =\int d \eta_{u_{1}} \ldots d \eta_{u_{m}}\left|\eta_{u_{i}}\right| \frac{\mathbf{e}^{-\sum_{j=1}^{m} \frac{\eta_{u_{j}}^{2}}{2 \sigma_{u_{u_{j}}}}}}{(2 \pi)^{m / 2} \Pi_{j=1}^{m} \sigma_{u_{j}}} \\
& =\sqrt{\frac{2}{\pi}} \sigma_{\eta_{u_{i}}},
\end{aligned}
$$

because $\sigma_{\eta_{u_{i}}}=\frac{\sigma_{1}}{\sqrt{m}}$ and $\sigma_{1}^{2} \equiv\left\langle\eta^{2}\right\rangle$, so $\left\langle\left|\eta_{u_{i}}\right|\right\rangle=\sqrt{\frac{2}{m \pi}} \sigma_{1}$. For $\langle|\eta|\rangle$, one can write

$$
\begin{aligned}
\langle|\eta|\rangle & =\int d \eta_{u_{1}} \ldots d \eta_{u_{m}}|\eta| \frac{e^{-\frac{\eta_{u_{u_{1}}}^{2}}{2 \sigma_{\eta_{u_{1}}}^{2}}-\frac{\eta_{u_{2}}^{2}}{2 \sigma_{\eta_{u_{2}}}^{2}} \cdots-\frac{\eta_{u_{m}}^{2}}{2 \sigma_{\eta_{u_{m}}}^{2}}}}{(2 \pi)^{m / 2} \sigma_{u_{1}} \ldots \sigma_{u_{m}}} \\
& =\sqrt{\frac{2}{m}} \frac{\Gamma\left(\frac{m+1}{2}\right)}{\Gamma\left(\frac{m}{2}\right)} \sigma_{1} .
\end{aligned}
$$

Subsequently, for $m=2:\left\langle\mid \eta_{\diamond}\right\rangle=\frac{2}{\pi}\langle|\eta|\rangle=\frac{\sigma_{1}}{\sqrt{\pi}}$. Plugging them in Eq. (9), one can simply get the theoretical prediction for the Gaussian rough surface in an arbitrary direction represented by Eq. (8). In the presence of weak nonGaussianity, there is a perturbative approach to set up the theoretical model for any desired feature (see also Ref. 56). Here to complete the explanation, we will consider the perturbative equations up to $\mathcal{O}\left(\sigma_{0}^{3}\right)$, for the up-crossing
[Eq. (9)]. The characteristic function related to the free energy of the underlying field is defined by ${ }^{56}$

$$
Z(\lambda)=\int_{-\infty}^{+\infty} d^{N} A \mathcal{P}(\mathbf{A}) \mathbf{e}^{\mathbf{i} \lambda \cdot \mathbf{A}}
$$

Here, A contains $N$ components. Using the definition of cumulants, $K_{\beta_{1}, \beta_{2}, \ldots, \beta_{n}}^{n} \equiv\left\langle A_{\beta_{1}} A_{\beta_{2}} \ldots A_{\beta_{n}}\right\rangle_{c}$ (here \langle\rangle$_{c}$ is written to emphasize that here we have cumulants rather than moments. As examples $\left\langle A_{\beta_{1}}\right\rangle_{c}=\langle\mathcal{H}\rangle_{c}$ and $\left\langle A_{\beta_{1}} A_{\beta_{1}}\right\rangle_{c}$ $=\left\langle\mathcal{H}^{2}\right\rangle_{c}=\left\langle\mathcal{H}^{2}\right\rangle-\langle\mathcal{H}\rangle^{2}$. If the mean value of the underlying stochastic field is zero, cumulants are identical to moments.), one can expand $\ln (Z(\lambda))$ as

$$
\ln (Z(\lambda))=\sum_{j=1}^{\infty} \frac{\mathbf{i}^{j}}{j !}\left(\sum_{\beta_{1}}^{N} \sum_{\beta_{2}}^{N} \ldots \sum_{\beta_{j}}^{N} K_{\beta_{1}, \beta_{2}, \ldots, \beta_{j}}^{j} \lambda_{\beta_{1}} \lambda_{\beta_{2}} \ldots \lambda_{\beta_{j}}\right) .
$$

Hence the above equation becomes

$$
\begin{aligned}
Z(\lambda)= & \mathbf{e}^{-\frac{1}{2} \lambda^{T} \cdot \mathcal{M}^{-1} \cdot \lambda} \\
& \times \mathbf{e}^{\sum_{j=3}^{\infty} \frac{j}{j !}\left(\sum_{\beta_{1}}^{N} \sum_{\beta_{2}}^{N} \ldots \sum_{\beta_{j}}^{N} K_{\beta_{1}, \beta_{2}, \ldots, \beta_{j}}^{j} \lambda_{\beta_{1}} \lambda_{\beta_{2}} \ldots \lambda_{\beta_{j}}\right) .} .
\end{aligned}
$$

By using an inverse Fourier Transform, one can read the probability density function as follows:

$$
\mathcal{P}(\mathbf{A})=\frac{1}{(2 \pi)^{N}} \int_{-\infty}^{+\infty} d^{N} \lambda Z(\lambda) \mathbf{e}^{-\mathbf{i} \lambda . \mathbf{A}} .
$$

Plugging Eq. (A12) in Eq. (A13), we find

$$
\begin{aligned}
\mathcal{P}(\mathbf{A})= & \mathbf{e}^{\left[\sum_{j=3}^{\infty} \frac{(-1)^{j}}{j !}\left(\sum_{\beta_{1}}^{N} \sum_{\beta_{2}}^{N} \ldots \sum_{\beta_{j}}^{N} K_{\beta_{1}, \beta_{2}, \ldots, \beta_{j} \overline{\partial A} \beta_{1} \frac{\partial^{j}}{\partial A_{\beta_{2}} \ldots \partial A_{\beta_{j}}}}^{j}\right)\right]} \\
& \times \sqrt{\frac{\operatorname{det} \mathcal{M}}{(2 \pi)^{N}} \mathbf{e}^{-\frac{1}{2}\left(\mathbf{A}^{T} \cdot \mathcal{M} \cdot \mathbf{A}\right)} .}
\end{aligned}
$$

Here, $\mathcal{M}$ is the inverse of covariance $N \times N$ matrix, and for $N=3$, it is the same as Eq. (7). The last term in the above equation equates to the multivariate Gaussian probability density function introduced in Eq. (6). By using Eq. (A14), the statistical average of a typical feature, $f$, in the general case reads ${ }^{56}$

$$
\langle f\rangle=\int_{-\infty}^{+\infty} d^{N} A \mathcal{P}(\mathbf{A}) f(\mathbf{A})
$$

By taking into account up to $\mathcal{O}\left(\sigma_{0}^{3}\right)$ in the context of the perturbative approach, the probability density function of $\mathcal{H}$ reads as

$$
\begin{aligned}
\mathcal{P}(\alpha)= & \left\langle\delta_{d}(\mathcal{H}-\alpha)\right\rangle_{\mathcal{H}} \\
& \times \frac{1}{\sqrt{2 \pi} \sigma_{0}} \mathbf{e}^{-\alpha^{2} / 2 \sigma_{0}^{2}}\left[1+B \sigma_{0}+C \sigma_{0}^{2}+\mathcal{O}\left(\sigma_{0}^{3}\right)\right]
\end{aligned}
$$

in which 


$$
\begin{gathered}
B \equiv \frac{S_{0}}{6}\left(\frac{\alpha^{3}}{\sigma_{0}^{3}}-3 \frac{\alpha}{\sigma_{0}}\right) \\
C \equiv \frac{K_{0}}{24} H_{4}\left(\frac{\alpha}{\sigma_{0}}\right)+\frac{S_{0}^{2}}{72} H_{6}\left(\frac{\alpha}{\sigma_{0}}\right), \\
S_{0} \equiv \frac{\left\langle\mathcal{H}^{3}\right\rangle_{c}}{\sigma_{0}^{4}} \\
K_{0} \equiv \frac{\left\langle\mathcal{H}^{4}\right\rangle_{c}}{\sigma_{0}^{6}}
\end{gathered}
$$

also $H_{4}\left(\alpha / \sigma_{0}\right)$ and $H_{6}\left(\alpha / \sigma_{0}\right)$ are probabilistic's Hermite polynomials of orders 4 and 6 , respectively. Now we are ready to compute the crossing statistics represented in Eq. (9) in $m \mathrm{D}$ :

$$
\begin{aligned}
\nu_{\diamond}^{+}(\alpha)= & \frac{1}{2 \pi} \frac{\sigma_{1}}{\sqrt{m} \sigma_{0}} \mathbf{e}^{-\alpha^{2} / 2 \sigma_{0}^{2}} \\
& \times\left[1+B \sigma_{0}+C \sigma_{0}^{2}+\mathcal{O}\left(\sigma_{0}^{3}\right)\right],
\end{aligned}
$$

where

$$
\begin{gathered}
B \equiv \frac{S_{0}}{6}\left(\frac{\alpha^{3}}{\sigma_{0}^{3}}-3 \frac{\alpha}{\sigma_{0}}\right)+\frac{S_{1}}{3} \frac{\alpha}{\sigma_{0}}, \\
S_{1} \equiv-\frac{3}{4} \frac{\left\langle\mathcal{H}^{2} \nabla^{2} \mathcal{H}\right\rangle}{\sigma_{0}^{2} \sigma_{1}^{2}}
\end{gathered}
$$

also

$$
\begin{gathered}
C \equiv \frac{S_{0}^{2}}{72} H_{6}\left(\frac{\alpha}{\sigma_{0}}\right)+\frac{K_{0}-S_{0} S_{1}}{24} H_{4}\left(\frac{\alpha}{\sigma_{0}}\right) \\
-\frac{1}{12}\left(K_{1}+\frac{S_{1}^{2}}{8}\right) H_{2}\left(\frac{\alpha}{\sigma_{0}}\right)-\frac{1}{8} K_{3}, \\
K_{1} \equiv \frac{\left\langle\mathcal{H}^{3} \nabla^{2} \mathcal{H}\right\rangle_{c}}{\sigma_{0}^{4} \sigma_{1}^{2}}, \\
K_{3} \equiv \frac{\left\langle|\nabla \mathcal{H}|^{4}\right\rangle_{c}}{2 \sigma_{0}^{2} \sigma_{1}^{4}} .
\end{gathered}
$$

${ }^{1}$ M. Sahimi, Heterogeneous Materials II (Springer, Berlin, 2003), Chaps. $5-8$.

${ }^{2}$ A.-L. Barabási and H. E. Stanley, Fractal Concepts in Surface Growth (Cambridge University Press, 1995), p. 366.

${ }^{3}$ R. M. Bradley and J. M. E. Harper, J. Vac. Sci. Technol., A 6, 2390 (1988).

${ }^{4}$ Y. P. Zhao, G. C. Wang, and T. M. Lu, Phys. Rev. B 58, 7300 (1998).

${ }^{5}$ Y. P. Zhao, H. N. Yang, G. C. Wang, and T. M. Lu, Phys. Rev. B 57, 1922 (1998).

${ }^{6}$ T. R. Thomas, B. G. Rosén, and N. Amini, Wear 232, 41 (1999).

${ }^{7}$ J. Villain, J. Phys. I France 1, 19-42 (1991).

${ }^{8}$ D. E. Wolf, Phys. Rev. Lett. 67, 1783 (1991).

${ }^{9}$ T. Kloss, L. Canet, and N. Wschebor, Phys. Rev. E 90, 062133 (2014).

${ }^{10}$ R. Kree, T. Yasseri, and A. K. Hartmann, Nucl. Instrum. Methods Phys., B 267, 1407 (2009).

${ }^{11}$ E. J. Heller and M. G. Lagally, Appl. Phys. Lett. 60, 2675 (1992).

${ }^{12}$ R. Maboudian, V. Bressler-Hill, K. Pond, X. S. Wang, P. M. Petroff, and W. H. Weinberg, Surf. Sci. 302, 269 (1994).

${ }^{13}$ C. Orme, M. D. Johnson, J. L. Sudijono, K. T. Leung, and B. G. Orr, Appl. Phys. Lett. 64, 860 (1994).
${ }^{14}$ M. A. Cotta, R. A. Hamm, T. W. Staley, S. N. G. Chu, L. R. Harriott, M. B. Panish, and H. Temkin, Phys. Rev. Lett. 70, 4106 (1993).

${ }^{15}$ R. L. Headrick, J. M. Baribeau, and Y. E. Strausser, Appl. Phys. Lett. 66, 96 (1995).

${ }^{16}$ M. T. Sinn, J. A. del Alamo, B. R. Bennett, K. Haberman, and F. G. Celii, J. Electron. Mater. 25, 313 (1996).

${ }^{17}$ N. E. Lee, D. G. Cahill, and J. E. Greene, Phys. Rev. B 53, 7876 (1996).

${ }^{18}$ E. Vivo, M. Nicoli, M. Engler, T. Michely, L. Vázquez, and R. Cuerno, Phys. Rev. B 86, 245427 (2012)

${ }^{19}$ E. Vivo, M. Nicoli, and R. Cuerno, Phys. Rev. E 86, 051611 (2012).

${ }^{20}$ G. Guillemot, M. Bigerelle, and Z. Kawaja, Scanning 36, 127 (2014).

${ }^{21}$ B. Schmittmann, G. Pruessner, and H. K. Janssen, Phys. Rev. E 73, 051603 (2006).

${ }^{22}$ E. Vivo, M. Nicoli, and R. Cuerno, Phys. Rev. E 89, 042407 (2014).

${ }^{23}$ A. K. Hartmann, R. Kree, U. Geyer, and M. Kölbel, Phys. Rev. B 65, 193403 (2002).

${ }^{24}$ E. O. Yewande, A. K. Hartmann, and R. Kree, Phys. Rev. B 71, 195405 (2005).

${ }^{25}$ E. O. Yewande, R. Kreean, and A. K. Hartmann, Phys. Rev. B 73, 115434 (2006).

${ }^{26}$ A. K. Hartmann, R. Kree, and T. Yasseri, J. Phys.: Condens. Matter 21, 224015 (2009).

${ }^{27}$ For the full description and documentation of all the versions of the model, see T. Yasseri, How to make nano-waves on solid surfaces, Saarbrücken, VDM Verlag, ISBN:978-3639292602 (2010).

${ }^{28}$ M. Ansari-Rad, S. M. Vaez Allaei, and M. Sahimi, Phys. Rev. E 85, 021121 (2012).

${ }^{29}$ J. Feder, Fractals (Plenum Press, New York and London, 1988).

${ }^{30}$ G. F. Gu and W. X. Zhou, Phys. Rev. E 74, 061104 (2006).

${ }^{31}$ J. W. Kantelhardt, e-print arXiv:0804.0747v1.

${ }^{32}$ C. Meneveau and K. R. Sreenivasan, Phys. Rev. Lett. 59, 1424 (1987).

${ }^{33}$ R. Benzi, G. Paladin, G. Parisi, and A. Vulpiani, J. Phys. A 17, 3521 (1984).

${ }^{34} \mathrm{D}$. Schertzer and S. Lovejoy, in Turbulence and Chaotic Phenomena in Fluids, edited by T. Tatsumi (North-Holland, Amsterdam, 1984).

${ }^{35}$ D. Schertzer and S. Lovejoy, J. Geophys. Res. 92, 9693, doi:10.1029/ JD092iD08p09693 (1987).

${ }^{36}$ D. Schertzer, S. Lovejoy, F. Schmitt, Y. Chigirinskaya, and D. Marsan, Fractals 05, 427 (1997).

${ }^{37}$ G. R. Jafari, S. M. Fazeli, F. Ghasemi, S. M. V. Allaei, M. R. R. Tabar, A. I. Zad, and G. Kave, Phys. Rev. Lett. 91, 226101 (2003).

${ }^{38}$ H. O. Peitgen, D. Saupe, M. F. Barnsley, Y. Fisher, and M. McGuire, The Science of Fractal Images (Springer, New York, NY, 1988).

${ }^{39}$ M. Ausloos and D. H. Berman, Proc. R. Soc. London, A 400, 331 (1985).

${ }^{40}$ H. Hamzehpour and M. Sahimi, Phys. Rev. E 73, 056121 (2006).

${ }^{41}$ V. Bakolas, Wear 254, 546-554 (2003).

${ }^{42}$ H. A. Makse, S. Havlin, M. Schwartz, and H. E. Stanley, Phys. Rev. E 53, 5445 (1996).

${ }^{43} \mathrm{M}$. Sahimi, Flow and Transport in Porous Media and Fractured Rock, 2nd ed. (Wiley-VCH, Weinheim, 2011), Chap. 5

${ }^{44}$ M. Sahimi and S. E. Tajer, Phys. Rev. E 71, 046301 (2005).

${ }^{45}$ M. A. Knackstedt, A. P. Sheppard, and W. V. Pinczewski, Phys. Rev. E 58, R6923 (1998) ; M. A. Knackstedt, A. P. Sheppard, and M. Sahimi, Adv. Water Resour. 24, 257 (2001).

${ }^{46}$ S. Habenicht, W. Bolse, K. P. Lieb, and K. Reimann, Phys. Rev. B 60, R2200 (1999).

${ }^{47}$ U. Valbusa, C. Boragno, and F. B. de Mongeot, J. Phys.: Condens. Matter 14, 8153 (2002).

${ }^{48}$ F. Frost, B. Ziberi, A. Schindler, and B. Rauschenbach, Appl. Phys. A: Mater. Sci. Process. 91, 551 (2008).

${ }^{49}$ R. Kree, T. Yasseri, and A. K. Hartmann, Nucl. Instrum. Methods Phys. B 267, 1403 (2009).

${ }^{50}$ T. Yasseri and R. Kree, Nucl. Instrum. Methods Phys. B 268, 2496 (2010).

${ }^{51}$ A. D. Maynard et al., Nature 444, 267 (2006).

${ }^{52}$ P. Sigmund, Phys. Rev. 184, 383 (1969).

${ }^{53}$ S. O. Rice, Bell Syst. Tech. J. 23, 282 (1944); 24, 46 (1945).

${ }^{54}$ B. S. Ryden, Astrophys. J. 333, 41 (1988).

${ }^{55}$ J. R. Bond and G. Efstathiou, Mon. Not. R. Astron. Soc. 226, 655 (1987).

${ }^{56}$ T. Matsubara, Astrophys. J. 584, 1 (2003).

${ }^{57}$ T. Matsubara, Phys. Rev. D 81, 083505 (2010).

${ }^{58}$ F. Shahbazi, S. Sobhanian, M. R. Rahimi Tabar, S. Khorram, G. R. Frootan, and H. Zadeh, J. Phys. A 36, 2517 (2003).

${ }^{59}$ M. S. Movahed and S. Khosravi, JCAP 1, 012 (2011).

${ }^{60}$ D. E. Newland, An Introduction to Random Vibrations, Spectral and Wavelet Analysis, 3rd ed. (Longman Scientific Technical, 1993). 
${ }^{61} \mathrm{P} . \mathrm{H}$. Brill, "A brief outline of the level crossing method in stochastic models (English and French)," CORS (Canadian Operational Research Society), Bulletin 34(4), 9-21 (2000).

${ }^{62} \mathrm{Z}$. Zhao and W. B. Wu, Stochastic Process. Appl. 117, 862-877 (2007).

${ }^{63}$ H. E. Hurst, Trans. Am. Soc. Civ. Eng. 116, 770 (1951).

${ }^{64}$ C. K. Peng, S. Buldyrev, A. Goldberger, S. Havlin, F. Sciortino, M. Simons, and H. E. Stanley, Nature 356, 168 (1992).

${ }^{65}$ C. K. Peng, S. V. Buldyrev, S. Havlin, M. Simons, H. E. Stanley, and A. L. Goldberger, Phys. Rev. E 49, 1685 (1994).

${ }^{66}$ J. W. Kantelhardt, S. A. Zschiegner, E. Koscielny-Bunde, S. Havlin, A. Bunde, and H. E. Stanley, Phys. A 316, 87 (2002).

${ }^{67}$ K. Hu, P. C. Ivanov, Z. Chen, P. Carpena, and H. E. Stanley, Phys. Rev. E 64, 011114 (2001).
${ }^{68}$ A. Arnéodo, N. Decoster, P. Kestener, and S. G. Roux, Adv. Imaging Electron Phys. 126, 1 (2003).

${ }^{69}$ S. G. Roux, A. Arneodo, and N. Decoster, Eur. Phys. J. B 15, 765 (2000); N. Decoster, S. G. Roux, and A. Arneodo, ibid. 15, 739 (2000); A. Arnedo, N. Decoster, and S. G. Roux, ibid. 15, 567 (2000).

${ }^{70}$ J. F. Muzy, E. Bacry, and A. Arneodo, Phys. Rev. Lett. 67, 3515 (1991).

${ }^{71}$ Z. R. Struzik and A. P. J. M. Siebes, Phys. A 309, 388 (2002).

${ }^{72}$ J. Arrault, A. Arneodo, A. Davis, and A. Marshak, Phys. Rev. Lett. 79, 75 (1997).

${ }^{73}$ J. W. Kantelhardt, H. E. Roman, and M. Greiner, Phys. A 220, 219 (1995).

${ }^{74}$ H. E. Roman, J. W. Kantelhardt, and M. Greiner, Europhys. Lett. 35, 641 (1996).

${ }^{75}$ M. Vahabi, G. R. Jafari, and S. M. S. Movahed, J. Stat. Mech. 2011, P11021 (2011). 